BULL. AUSTRAL. MATH. SOC.

VOL. $29(1984), 91-98$.

\title{
SOME DELAYS DO NOT MATTER
}

\author{
K. Gopalsamy
}

\begin{abstract}
Sufficient conditions are derived for the solutions of a system of linear delay-differential equations to behave asymptotically as those of the same system without the argument delays. A generalisation of the result to integrodifferential systems is indicated.
\end{abstract}

\section{Introduction}

We consider an autonomous linear system of delay-differential equations

(1.1) $\frac{d x_{i}(t)}{d t}=a_{i i} x_{i}(t)+\sum_{\substack{j=1 \\ j \neq i}}^{n} a_{i j} x_{j}(t-\tau, j), i=1,2, \ldots n, \quad(n \geq 2)$

and a related system of differential equations with no argument-delays (1.2) $\frac{d y_{i}(t)}{d t}=a_{i i} y_{i}(t)+\sum_{\substack{j=1 \\ j \neq i}}^{n} a_{i j} y_{j}(t), i=1,2, \ldots, n ; \quad(n \geq 2)$ where $a_{i j} \tau_{i j}(i, j=1,2, \ldots, n)$ are real constants. It is of some interest to examine under what conditions, the solutions of the systems (1.1) and (1.2) are asymptotically equivalent in the sense that

$$
\lim _{t \rightarrow \infty}\left|x_{i}(t)-y_{i}(t)\right|=0 ; i=1,2, \ldots, n
$$

Received 13 February 1984.

Copyright Clearance Centre, Inc. Serial-fee code: 0004-9727/84 $\$ A 2.00+0.00$. 
where $x(t)=\left\{x_{1}(t), \ldots, x_{n}(t)\right\}$ and $y(t)=\left\{y_{1}(t), \ldots, y_{n}(t)\right\}$ are arbitrary solutions of (1.1) and (1.2) respectively. A number of authors (Cooke [1], Kato [3], Hale [2]) have discussed the problem of asymptotic (as $t \rightarrow \infty$ ) equivalence of solutions of differential-differeñce (or functional differential) equations and ordinary differential equations assuming that the relevant delays involved are small in some sense or the other. In the following we make no assumptions regarding the magnitudes of the argument delays $\tau_{i j}$ in (1.1) restricting ourselves to nonscalar systems.

\section{Asymptotic equivalence}

We will use the following notation; for any $x=\left(x_{1}, \ldots x_{n}\right) \in \mathbb{R}^{n}$, we define $\|x\|=\sum_{i=1}^{n}\left|x_{i}\right|$ and this vector norm induces a matrix norm defined by the following:

$$
\text { for } A=\left(a_{i j}\right) \quad(i, j=1,2, \ldots, n), \quad A \in \mathbb{R}^{n \times n}
$$

$$
\|A\|=\max _{j} \sum_{i=1}^{n}\left|a_{i j}\right| .
$$

If $A$ is any real $n \times n$ matrix then a measure of the matrix denoted by $\mu(A)$ is defined as follows:

$$
\mu(A)=\lim _{\theta \rightarrow 0+}(\|I+\theta A\|-1) / \theta
$$

where $I$ denotes the $n \times n$ identity matrix. It is known that for the norm in (2.1), $\mu(A)$ is given by

$$
\mu(A)=\max _{j}\left[a_{j j}+\sum_{\substack{i=1 \\ i \neq j}}^{n}\left|a_{i j}\right|\right] .
$$

We will first derive the following: 
LEMMA 2.1. If

$$
\mu(A) \leq-\alpha<0 \text { for some constant } \alpha>0
$$

then all solutions of (1.1) corresponding to initial conditions of the form

$$
x_{i}(s)=\phi_{i}(s), \phi_{i} \in C([-\tau, 0], \mathbb{R}), \tau=\max _{\substack{1 \leq i, j \leq n \\ i \neq j}} \tau i j
$$

are such that

$$
\begin{aligned}
& \text { (2.6) (i) }\|x(t)\|=\sum_{i=1}^{n}\left|x_{i}(t)\right| \text { is bounded for } t \in[0, \infty) \\
& \text { (2.7) (ii) } \int_{0}^{\infty}\|x(t)\| d t<\infty .
\end{aligned}
$$

Proof. If $z$ is any continuously differentiable scalar function on $(0, \infty)$, define a functional $\sigma$ as follows:

$$
\sigma(z)(t)=\left\{\begin{array}{cc}
1 & \text { if } z(t)>0 \text { or } z(t)=0 \text { and } \frac{d z(t)}{d t}>0 \\
0 & \text { if } z(t)=0 \text { and } \frac{d z(t)}{d t}=0 \\
-1 \text { if } z(t)<0 \text { or } z(t)=0 \text { and } \frac{d z(t)}{d t}<0 .
\end{array}\right.
$$

It can be found from (2.8) that

$$
\sigma(z)(t) z(t)=|z(t)| \text { and } \sigma(z)(t) \frac{d z(t)}{d t}=D^{+}|z(t)|
$$

where $D^{+}|z(t)|$ denotes the upper right Dini-derivative of $|z(t)|$. Consider a Lyaponov functional $v(t)=v\left(t, x_{1}(t), \ldots, x_{n}(t)\right)$ defined by

$$
v(t)=\sum_{i=1}^{n}\left\{\left|x_{i}(t)\right|+\sum_{\substack{j=1 \\ j \neq i}}\left|a_{i j}\right| \int_{t-\tau} \int_{i j}^{t}\left|x_{j}(s)\right| d s\right\} ; t \geq 0 .
$$

It is immediate from (2.10) that

$$
v(t) \geq\|x(t)\| \text { for } t \geq 0 \text { and } v(0)<\infty \text {. }
$$

Calculating the upper right Dini-derivative $D^{+} V(t)$ along the solutions of (1.1), we have 
(2.12) $D^{+} v(t)=\sum_{i=1}^{n}\left\{\sigma\left(x_{i}\right)(t) \frac{d x_{i}(t)}{d t}+\sum_{\substack{j=1 \\ j \neq i}}^{n}\left|a_{i j}\right|\left[\left|x_{j}(t)\right|-\left|x_{j}\left(t-\tau{ }_{i j}\right)\right|\right]\right\}$ and simplifying (2.12) using (2.9) and (2.4),

$$
\begin{aligned}
D^{+} v(t) & \leq \sum_{i=1}^{n}\left\{a_{i i}\left|x_{i}(t)\right|+\sum_{\substack{j=1 \\
j \neq i}}^{n}\left|a_{i j}\right|\left|x_{j}(t)\right|\right\} \\
& \leq \sum_{i=1}^{n}\left[a_{i i}+\sum_{\substack{j=1 \\
j \neq i}}^{n}\left|a_{j i}\right|\right]\left|x_{i}(t)\right| \\
& \leq \mu(A)\|x(t)\| \leq-\alpha\|x(t)\| .
\end{aligned}
$$

(Note that $(2.4)$ implies, $\left.a_{i i}<0, i=1,2, \ldots, n\right)$. An integration of (2.13) along with (2.11) leads to

$$
v(t)+\alpha \int_{0}^{t}\|x(s)\| d s \leq v(0)<\infty
$$

and hence

$$
\|x(t)\|+\alpha \int_{0}^{t}\|x(s)\| d s \leq v(0)<\infty
$$

Since $\alpha>0$, the result follows from (2.14).

Our principal result is the following:

THEOREM 2.1. Assume that the conditions of the above lemma hold; then if $x(t)$ and $y(t)$ are arbitrary solutions of (1.1) and (1.2) respectively then

$$
\lim _{t \rightarrow \infty}\left|x_{i}(t)-y_{i}(t)\right|=0 ; i=1,2, \ldots, n .
$$

Proof. It is found from (1.1) and (1.2) that

$$
\frac{d}{d t}\left[x_{i}(t)-y_{i}(t)\right]=a_{i i}\left[x_{i}(t)-y_{i}(t)\right]+\sum_{\substack{j=1 \\ j \neq i}}^{n} a_{i j}\left[x_{j}(t)-y_{j}(t)\right]
$$

$$
\begin{aligned}
-\sum_{\substack{j=1 \\
j \neq i}}^{n} a_{i j}\left[x_{j}(t)-x_{j}(t-\tau, j)\right] & \\
i & =1,2, \ldots, n .
\end{aligned}
$$


Some delays do not matter

As in the lemma, we can derive

$$
\begin{aligned}
D^{+}\left[\sum_{i=1}^{n}\left|x_{i}(t)-y_{i}(t)\right|\right] \leq & \mu(A) \sum_{i=1}^{n}\left|x_{i}(t)-y_{i}(t)\right| \\
& +\sum_{i=1}^{n} \sum_{\substack{1 \neq j \\
j=1}}^{n}\left|a_{i j}\right|\left[\left|x_{j}(t)\right|+\left|x_{j}\left(t-\tau_{i j}\right)\right|\right] .
\end{aligned}
$$

Thus we have

$$
\begin{aligned}
D^{+}\|x(t)-y(t)\| & \leq-\alpha\|x(t)-y(t)\|+\sum_{\substack{i, j=1 \\
i \neq j}}^{n}\left|\sum_{i j}\right|\left[\|x(t)\|+\left\|x\left(t-\tau_{i j}\right)\right\|\right] \\
& \leq-\alpha\|x(t)-y(t)\|+n\|A\|\left[\|x(t)\|+\left\|x\left(t-\tau_{i j}\right)\right\|\right]
\end{aligned}
$$

which implies on integration,

$$
\begin{aligned}
\|x(t)-y(t)\|+ & \alpha \int_{0}^{t}\|x(s)-y(s)\| d s \leq\|x(0)-y(0)\| \\
& +2 n\|A\| \int_{0}^{\infty}\|x(s)\| d s+n A \int_{-\tau}^{0}\|x(s)\| d s \\
& <\infty \quad \text { (by lemma 2.1). }
\end{aligned}
$$

A consequence of $(2.18)$ is that $\|x(t)-y(t)\|$ is bounded for $t \in[0, \infty)$ which together with the boundedness of $\|x(t)\|, t \in[-\tau, \infty)$ will imply that the right side of $(2.16)$ is bounded for $t \in[0, \infty)$ and hence we note that $\|x(t)-y(t)\|$ is uniformly continuous for $t \in(0, \infty)$. (2.18) also implies that $\|x(\cdot)-y(\cdot)\| \in L_{1}(0, \infty)$ since $\|x(\cdot)\| \in L_{1}(0, \infty)$ by lemma 2.1. Such a uniform continuity of $\|x(\cdot)-y(\cdot)\|$ on $[0, \infty)$ together with the fact that $\|x(\cdot)-y(\cdot)\| \in L_{1}(0, \infty)$ implies $\|x(t)-y(t)\| \rightarrow 0$ as $t \rightarrow \infty$ and the proof is complete.

\section{Equations with unbounded delays}

We will briefly indicate here how the conclusions of lemma 2.1 and theorem 2.1 can be generalised to a class of integrodifferential equations (or equations with "unbounded delays"). For instance we will consider the solutions of 
$(3.1)$

$$
\begin{aligned}
& \frac{d x_{i}(t)}{d t}=a_{i i} x_{i}(t)+\sum_{j=1}^{n} a_{i j} \int_{0}^{\infty} k_{i j}(s) x_{j}(t-s) d s \\
& j \neq i \\
& i=1,2, \ldots, n
\end{aligned}
$$

under the following hypotheses:

(i) the system (3.1) is supplemented with initial conditions of the form

(3.2) $x_{i}(s)=\phi_{i}(s), s \in(-\infty, 0] ; \phi_{i}$ is bounded and continuous on $(-\infty, 0] ; i=1,2, \ldots, n$.

(ii) the delay kernels $k_{i j}:[0, \infty) \rightarrow(-\infty, \infty), i, j=1,2, \ldots, n ; i \neq j$ are piecewise (locally) continuous on $[0, \infty)$ such that

$$
\begin{aligned}
& \int_{0}^{\infty}\left|k_{i j}(s)\right| d s<\infty ; \int_{0}^{\infty} s\left|k_{i j}(s)\right| d s<\infty \\
& i, j=1,2, \ldots, n ; \quad i \neq j .
\end{aligned}
$$

(iii) $a_{i j}(i, j=1,2, \ldots, n)$ are real constants such that

$$
\begin{gathered}
a_{i i}<0 ; i=1,2, \ldots, n \\
\mu(A ; k)=\max _{j} \cdot\left[a_{j j}+\sum_{\substack{i=1 \\
i \neq j}}^{n}\left|a_{i j}\right| \int_{0}^{\infty}\left|k_{i j}(s)\right| d s\right] \leq-\alpha<0 \\
\text { for some constant } \alpha>0
\end{gathered}
$$

Analogous to lemma 2.1 we have the following:

LEMMA 3.1. Assume that the above hypotheses (i) - (iii) hold; then all solutions of (3.1) corresponding to initial conditions in (3.2) are such that

(a) $\sum_{i=1}^{n}\left|x_{i}(t)\right|$ is bounded for $t \in[0, \infty)$

$$
\text { (b) } \int_{0}^{\infty}\left(\sum_{i=1}^{n}\left|x_{i}(t)\right|\right) d t<\infty \text {. }
$$

Proof. We consider a Lyapunov functional $v(t)$ defined by

$$
v(t)=\sum_{i=1}^{n}\left[\left|x_{i}(t)\right|+\sum_{\substack{j=1 \\ j \neq i}}^{n}\left|a_{i j}\right| \int_{0}^{\infty}\left|k_{i j}(s)\right|\left(\int_{t-s}^{t}\left|x_{j}(u)\right| d u\right) d s\right]
$$


and note that

$$
v(t) \geq \sum_{i=1}^{n}\left|x_{i}(t)\right|
$$

and

$$
\begin{aligned}
v(0) & \left.=\sum_{i=1}^{n}\left[\left|x_{i}(0)\right|+\sum_{\substack{j=1 \\
j \neq i}}^{n}\left|a_{i j}\right|\left\{\int_{0}^{\infty}\left|k_{i j}(s)\right| \iint_{-s}^{0}\left|x_{j}(u)\right| d u\right) d s\right\}\right] \\
& \leq \sum_{i=1}^{n}\left[\left|x_{i}(0)\right|+\sum_{\substack{j=1 \\
j \neq i}}^{n}\left|a_{i j}\right|\left(\sup _{u \leq 0}\left|x_{j}(u)\right|\right) \int_{0}^{\infty} s k_{i j}(s) \mid d s\right]
\end{aligned}
$$

$$
<\infty \text { (by hypotheses (i) and (ii)). }
$$

Since the remainder of the proof is similar to that of lemma 2.1 , we will omit the details.

The proof of the following is similar to that of theorem 2.1 if one uses the conclusion of lemma 3.1 and hence we will suppress its proof.

THEOREM 3.1. Assume that the hypotheses (i) - (iii) above hold; then if $y(t)=\left(y_{1}(t), \ldots y_{n}(t)\right)$ is any solution of the system

$$
\frac{d y_{i}(t)}{d t}=a_{i i} y_{i}(t)+\sum_{\substack{j=1 \\ j \neq i}}^{n} a_{i j}\left(\int_{0}^{\infty} k_{i j}(s) d s\right) y_{j}(t)
$$

we have

$$
\lim _{t \rightarrow \infty}\left|x_{i}(t)-y_{i}(t)\right|=0 ; \quad i=1,2, \ldots, n
$$

where $x(t)=\left(x_{1}(t) \ldots x_{n}(t)\right)$ is any solution of (3.1).

We conclude with the following remarks:

1. If $x(t)=\left\langle x_{1}(t), x_{2}(t), \ldots, x_{n}(t)\right), t \in[-\tau, \infty)$ is any solution of

(1.1) then there exists a unique solution $y(t)=\left(y_{1}(t), \ldots, y_{n}(t)\right)$,

$t \in[0, \infty)$ of $(1.2)$ satisfying $x(0)=y(0)$ and

$\|x(t)-y(t)\| \rightarrow 0$ as $t \rightarrow \infty$; and conversely for any solution $y(t)$ of 
(1.2) there exists a unique solution $x(t)$ of (1.1) satisfying $x(s) \equiv y(0), s \in[-\tau, 0]$ and $\|x(t)-y(t)\| \rightarrow 0$ as $t \rightarrow \infty$.

2. The delays $\tau_{i j}(i \neq j, i, j=1,2, \ldots, n)$ need not be small in any sense for the validity of our results.

\section{References}

[1] K.L. Cooke, "Functional differential equations with asymptotically vanishing lag", Rend. Circ. Mat. Palermo. Ser. II 16 (1967), 39-56.

[2] J.K. Hale, "Linear asymptotically autonomous functional differential equations. Rend. Circ. Math. Palermo. Ser. II 15 (1966), 331-351.

[3] J. Kato, "On the existence of a solution approaching zero for functional differential equations", Proceedings of the U.S.Japan Seminar on differential and functional equations", 153-169 Benjamin, New York 1967.

School of Mathematical Sciences,

The Flinders University of South Australia,

Bedford Park, S.A. 5042,

Australia. 